\title{
Adaptive Optimizing Nonlinear Control Design for an Over-actuated Aircraft Model
}

\author{
E.R. van Oort; L. Sonneveldt† Q.P. Chu $\ddagger$ J.A. Mulder ${ }^{\S}$ \\ Delft University of Technology, P.O. Box 5058, 2600 GB Delft, The Netherlands
}

\begin{abstract}
In this paper nonlinear adaptive flight control laws based on the backstepping approach are proposed which are applicable to over-actuated nonlinear systems. Instead of solving the control allocation exactly, update laws for the desired control effector signals are defined such that they converge to the optimal solution. Stability and boundedness of the resulting closed-loop system can be shown by means of Lyapunov analysis. Three different update laws are defined, the integrated, modular and composite adaptive designs. The last can be seen as a combination between the first two and has the best convergence and numerical properties. Second-order actuator dynamics are taken into account in the control designs. The control design is evaluated using numerical simulations where several cases of locked control surface failures are considered during two different maneuvers. No sensor information about these failures is fed back to the control system. The tracking performance of the adaptive control design is excellent for the nominal case and all considered failure cases. The failures are recognized shortly after they are introduced into the system, and the new dynamics are rapidly identified.
\end{abstract}

\section{Nomenclature}

$\alpha$

$\alpha_{0}$

$\beta$

$\Delta \alpha$

$\delta_{\star}$

$\hat{\theta}$

$\phi$

$\theta$

$\varphi$

$\vartheta$

$g_{0}$

$i_{\star}$

$l_{\star}$

$m_{\star}$

$n_{\star}$

$p$

$q$

$r$

V

$y_{\star}, z_{\star}$ AOCLF

OCLF
Angle of attack

Trim angle of attack

Sideslip angle

Difference between $\alpha$ and $\alpha_{0}$

Control surface deflection

Estimated parameter

Roll angle

Parameter vector

Regressor function

Pitch angle

Gravitational acceleration constant

Inertia parameter

Roll moment component, dependent on $\star$

Pitch moment component, dependent on $\star$

Yaw moment component, dependent on $\star$

Roll rate

Pitch rate

Yaw rate

Velocity

Aerodynamic force component in $y, z$-body direction, dependent on $\star$

Adaptive Optimizing Control Lyapunov Function

Optimizing Control Lyapunov Function rad

rad

rad

rad

rad

rad

$\mathrm{rad}$

$\mathrm{m} / \mathrm{s}^{2}$

$\mathrm{rad} / \mathrm{s}$

$\mathrm{rad} / \mathrm{s}$

$\mathrm{rad} / \mathrm{s}$

$\mathrm{m} / \mathrm{s}$

*PhD Student, Control and Simulation Division, e.r.vanoort@tudelft.nl, AIAA Student Member.

$\dagger$ PhD Student, Control and Simulation Division, AIAA Student Member.

$¥$ Associate Professor, Control and Simulation Division, Faculty of Aerospace Engineering, AIAA Member.

$\S$ Professor, Control and Simulation Division, Faculty of Aerospace Engineering, AIAA Member. 


\section{Introduction}

Nearly all modern aircraft, ranging from general aviation aircraft to commercial aircraft and military jets, have become over-actuated systems. While this facilitates on-line reconfiguration, it creates an additional problem for the control engineer. The main question to be answered is, what is the best way to use the available control effectors to realize the desired control effort? Hence, some kind of cost function should be formulated and optimized subject to the constraint that the total control effort is equal, or as close as possible, to the desired control effort, such that the aircraft handles as intended by the designer. As shown in Ref. 1, the choice of control allocation method can have drastic effects on the performance of (nonlinear) control designs, especially for adaptive designs. In Ref. 2 a comparison was made for three different adaptive backstepping designs, with two different explicit control allocation approaches. It is possible to proof closed loop boundedness of the augmented tracking errors for these designs.

Optimizing control allocation solutions have been derived for certain classes of over-actuated systems, including aircraft, automotive vehicles and marine vessels. ${ }^{3,4,5,6,7,8,9,10,11,12}$ In many of these works, the control allocation problem is viewed as a static or quasi-dynamic optimization problem which is solved independently of the dynamic control problem. It is not necessary to solve the optimization problem exactly at each time instant ${ }^{11}$. The main advantage of this approach is computational efficiency and simple implementation. Furthermore, the method is able to handle non-affine cost functions and control effort mappings when convexity constraints are satisfied. Stability of the closed-loop system is shown through Lyapunov-analysis of a time-varying optimal set composed of the tracking errors, estimation error and first-order derivatives of a Lagrangian cost function.

The paper is outlined as follows. First of all the problem is presented in mathematical form and some preliminaries are discussed. Afterwards, the non-adaptive high level control with dynamic control allocation scheme is introduced, and its stability and convergence results are analyzed. Then, the non-adaptive control design is extended in three different adaptive designs: integrated, modular, and composite. The composite adaptive approach is applied to the control design of the simple over-actuated fighter aircraft model. Simulation results for two specific failure cases are demonstrated. Finally, the conclusions are presented and recommendations for future research formulated.

\section{Problem Definition and Preliminaries}

Consider a system in the form

$$
\begin{aligned}
\dot{x}_{1} & =f_{1}(t, x, u)+\varphi_{1}^{T}(t, x, u) \theta+g_{1}(t, x, u) x_{2} \\
\dot{x}_{2} & =f_{2}(t, x, u)+\varphi_{2}^{T}(t, x, u) \theta+g_{2}(t, x, u) x_{3} \\
& \vdots \\
\dot{x}_{n-1} & =f_{n-1}(t, x, u)+\varphi_{n-1}^{T}(t, x, u) \theta+g_{n-1}(t, x, u) x_{n} \\
\dot{x}_{n} & =f_{n}(t, x)+\varphi_{n}^{T}(t, x) \theta+g_{n}(t, x) \tau,
\end{aligned}
$$

with a static mapping from the control effectors $u$ to the virtual controls $\tau$

$$
\tau=h(t, x, u, \theta)=\varphi_{u}^{T}(t, x, u) \theta+\varphi_{0}^{T}(t, x, u) .
$$

Then, the system dynamics can be written in alternative form as $\dot{x}=G(t, x, u, \theta)$. Furthermore, there are actuator dynamics between the control laws and the actual system. The commanded control effector signals $u_{d}$ are filtered by the actuator model to produce the actual input to the system $u$. This is modeled as

$$
\dot{u}=m\left(t, x, u, u_{d}\right)
$$

where the function $m$ is assumed to be known, measurements of $u$ are not required by the controller. Additionally, the following assumption is made on the system (1).

Assumption II.1 The function $h$ is twice differentiable, and there exist constants $0<\rho_{1}<\rho_{2}$, such that $\forall t, x, u, \theta$

$$
\rho_{1} I<\frac{\partial h}{\partial u}(t, x, u, \theta)\left(\frac{\partial h}{\partial u}(t, x, u, \theta)\right)^{T} \leq \rho_{2} I .
$$


This assumption can be viewed as a controllability assumption in the sense that the mapping $h$ is surjective, and there exists a function $f_{u}(t, x, \theta)$ such that $h\left(t, x, f_{u}(t, x, \theta), \theta\right)=\tau_{d}$ for all $t, x, \theta$, where $\tau_{d}$ is the desired control effort. The problem of finding the optimal control allocation is formulated as a static minimization problem as

$$
\min _{u_{d}} J\left(t, x, u_{d}\right) \quad \text { subject to } \quad \tau_{d}-h\left(t, x, u_{d}, \theta\right)=0 .
$$

where $J$ is a cost function that incorporates objectives such as minimum power consumption, wear and tear, effects related to actuator configuration (singularity avoidance), and actuator constraints. Based on the optimization problem, the Lagrangian function

$$
L\left(t, x, u_{d}, \lambda, \hat{\theta}\right)=J\left(t, x, u_{d}\right)+\left(h\left(t, x, u_{d}, \hat{\theta}\right)-\tau_{d}\right)^{T} \lambda
$$

is introduced. The assumptions made on the cost function are

Assumption II.2 (Cost Function Assumptions)

1. The cost function $J$ is twice differentiable and $J\left(t, x, u_{d}\right) \rightarrow \infty$ as $\left|u_{d}\right| \rightarrow \infty$. Furthermore, $\frac{\partial J}{\partial u_{d}}, \frac{\partial^{2} J}{\partial t \partial u_{d}}$ and $\frac{\partial^{2} J}{\partial x \partial u_{d}}$ are uniformly bounded by $x$ and $u_{d}$.

2. There exists constants $0<k_{1}<k_{2}$ such that $\forall t, x, \theta$ and $\left(u_{d}^{T} \lambda^{T}\right)^{T} \notin \mathcal{O}_{u \lambda}^{0}$, where $\mathcal{O}_{u \lambda}=\left\{u_{d}^{T}, \lambda^{T} \mid \frac{\partial L}{\partial u_{d}}=0, \frac{\partial L}{\partial \lambda}=0\right\}$

$$
k_{1} I<\frac{\partial^{2} L}{\partial u^{2}}\left(t, x, u_{d}, \lambda, \theta\right) \leq k_{2} I,
$$

where $\mathcal{O}_{u \lambda}^{0}$ is the interior of $\mathcal{O}_{u \lambda}$. In the interior, the lower bound is replaced by $\frac{\partial^{2} L}{\partial u} \geq 0$.

The second order sufficient conditions are satisfied for all $t, x, u, \lambda$ and $\theta$ by these assumptions. ${ }^{13}$ Hence the set $\mathcal{O}_{u \lambda}$ describes global optimal solutions of the problem (5). The control problem is to design a controller which is able to track a reference signal $y_{r}(t) \in \mathbb{C}^{n}$ asymptotically when the parameters $\theta$ are not known, and simultaneously optimize the cost function $J$.

\section{Non-adaptive Control Design}

In this section the adaptive optimizing control design is introduced. First of all the combination of backstepping with optimizing dynamic control allocation approach is derived when the system is completely known. The steps up until the final step are identical to the normal backstepping design, where a virtual control law for the desired control effort $\tau_{d}$ is derived. The task of control allocation is to generate commands for the control effectors such that the desired control effort is generated and the cost function is minimized. Closed-loop stability and convergence to the optimal solution can then be shown through Lyapunov analysis.

\section{III.A. High-level Control Design}

The backstepping design is performed using CLFs of the form

$$
V_{i+1}\left(\bar{z}_{1}, \ldots, \bar{z}_{i}\right)=V_{i}+\frac{1}{2} \bar{z}_{i}^{T} \bar{z}_{i}
$$

where $\bar{z}_{i}$ are the augmented tracking errors. More detail and extensive discussion of the adaptive backstepping approach for aerospace applications can be found in papers such as Refs. 14,15,16. The backstepping design is ends with a virtual control law for the desired total control effort $\tau$. The resulting backstepping control design can be summarized as

$$
\begin{aligned}
z_{i} & =x_{i}-x_{i_{c}} \\
\bar{z}_{i} & =z_{i}-\Xi_{i} \\
\dot{\Xi}_{i} & =-C_{1} \Xi_{i}+g_{i}\left(x_{i+1_{c}}-x_{i+1_{c}}^{0}\right) \quad \text { for } i=1, \ldots, n-1 \\
x_{i_{c}}^{0} & =\alpha_{i-1}-\Xi_{i} \text { for } i=2, \ldots, n \\
\alpha_{i} & =g_{i}^{-1}\left(-f_{i}-\varphi_{i}^{T} \hat{\theta}-C_{i} z_{i}-g_{i-1}^{T} \bar{z}_{i-1}+\dot{x}_{i_{c}}\right)
\end{aligned}
$$


where the desired commands $x_{i_{c}}^{0}$ are filtered to produce $x_{i_{c}}$ and $\dot{x}_{i_{c}}$, and the desired control effort $\tau_{d}=\alpha_{n}$. If the generated control effort $\tau$ is equal to the desired total control effort $\tau_{d}$, the CLF derivative at the final step becomes

$$
\dot{V}_{n}=-\sum_{i=1}^{n} \bar{z}_{i}^{T} C_{i} \bar{z}_{i} \leq 0 \forall \bar{z} \neq 0
$$

showing UGAS of the equilibrium $\bar{z}=0$, which for appropriate filter settings can be used to show stability and convergence of the tracking error $z$ to zero.

\section{III.B. Control Allocation Design}

The task of the control allocation design is to transform the desired virtual control commands $\tau_{d}$ to actual control effector commands $u_{d}$ based on the solution of the optimization problem (5). This will be done by considering first-order optimality of the Lagrangian function (6). Update laws for the effector reference commands $u_{d}$ and the Lagrangian parameter $\lambda$ are then defined, such that $u_{d}$ and $\lambda$ converge to a set defined by the time-varying optimality condition. Stability and convergence of the closed loop system is shown for the time and state varying optimal set $\mathcal{A}=\left\{\left(\bar{z}^{T} u^{T} \lambda^{T}\right)^{T} \mid\left(\bar{z}^{T} \frac{\partial L^{T}}{\partial u} \frac{\partial L^{T}}{\partial \lambda}\right)^{T}=0\right\}$. The design of the update laws is based on the following optimizing control Lyapunov function (OCLF)

$$
V_{\text {ca }}\left(t, \bar{z}, \hat{\theta}, u_{d}, \lambda\right)=\sigma V_{n}(t, \bar{z}, \hat{\theta})+\frac{1}{2}\left(\frac{\partial L^{T}}{\partial u_{d}} \frac{\partial L}{\partial u_{d}}+\frac{\partial L^{T}}{\partial \lambda} \frac{\partial L}{\partial \lambda}\right),
$$

which is an extension of the CLF (8) with terms penalizing the first order derivatives of the Lagrangian function with respect to the desired control effector signals, and the Lagrangian parameter. The parameter $\sigma>0$ is an arbitrary design constant used to control the relative weight of the tracking error subsystem in the OCLF. The time derivative of the OCLF along the trajectories of the system (1) with the control effector mapping (2) is given by

$$
\begin{aligned}
\dot{V}_{\mathrm{ca}}= & \sigma\left(\frac{\partial V_{n}}{\partial t}+\frac{\partial V_{n}^{T}}{\partial \bar{z}}\left(G(t, x, u, \hat{\theta})-\dot{x}_{c}-\dot{\Xi}\right)\right) \\
& +\left(\frac{\partial L^{T}}{\partial u_{d}} \frac{\partial^{2} L}{\partial u_{d}^{2}}+\frac{\partial L}{\partial \lambda} \frac{\partial^{2} L}{\partial u_{d} \partial \lambda}\right) \dot{u}_{d}+\frac{\partial L^{T}}{\partial u_{d}} \frac{\partial^{2} L}{\partial \lambda \partial u_{d}} \dot{\lambda} \\
& +\left(\frac{\partial L^{T}}{\partial u_{d}} \frac{\partial^{2} L}{\partial \bar{z} \partial u_{d}}+\frac{\partial L^{T}}{\partial \lambda} \frac{\partial^{2} L}{\partial \bar{z} \partial \lambda}\right) \dot{\bar{z}}+\frac{\partial L^{T}}{\partial u_{d}} \frac{\partial^{2} L}{\partial t \partial u_{d}}+\frac{\partial L^{T}}{\partial \lambda} \frac{\partial^{2} L}{\partial t \partial \lambda} \\
= & -\sigma \sum_{i=1}^{n} \bar{z}_{i}^{T} C_{i} \bar{z}_{i}+\left[\begin{array}{c}
\frac{\partial L}{\partial u_{d}} \\
\frac{\partial L}{\partial \lambda}
\end{array}\right]^{T} H\left[\begin{array}{c}
\dot{u}_{d} \\
\dot{\lambda}
\end{array}\right]+\left[\begin{array}{c}
\frac{\partial L}{\partial u_{d}} \\
\frac{\partial L}{\partial \lambda}
\end{array}\right]^{T} u_{\mathrm{ff}}
\end{aligned}
$$

where

$$
H=\left[\begin{array}{cc}
\frac{\partial^{2} L}{\partial u_{d}^{2}} & \frac{\partial^{2} L}{\partial \lambda \partial u_{d}} \\
\frac{\partial^{2} L}{\partial u_{d} \partial \lambda} & 0
\end{array}\right],
$$

$x_{c}$ is composed of $y_{r}$ and the $x_{i_{c}}$, and, when $\operatorname{det}\left(\frac{\partial^{2} L}{\partial u_{d}^{2}}\right) \geq \varsigma$

$$
u_{\mathrm{ff}}=\left[\begin{array}{c}
\frac{\partial^{2} L}{\partial t \partial u_{d}} \\
\frac{\partial^{2} L}{\partial t \partial \lambda}
\end{array}\right]+\left[\begin{array}{c}
\frac{\partial^{2} L}{\partial \bar{z} \partial u_{d}} \\
\frac{\partial^{2} L}{\partial \bar{z} \partial \lambda}
\end{array}\right]\left(G(t, x, u, \hat{\theta})-\dot{x}_{c}-\dot{\Xi}\right),
$$

else $u_{f f}=0$. The update laws for $u_{d}$ and $\lambda$ now follow straightforward from the OCLF derivative, where they are used to cancel the known parts related to the cost function partial derivatives:

$$
\left[\begin{array}{c}
\dot{u}_{d} \\
\dot{\lambda}
\end{array}\right]=-\Gamma_{\mathrm{ca}} H\left[\begin{array}{c}
\frac{\partial L}{\partial u_{d}} \\
\frac{\partial L}{\partial \lambda}
\end{array}\right]-H^{-1} u_{\mathrm{ff}} .
$$


where $\Gamma_{\mathrm{ca}}$ is a symmetric, positive definite gain matrix. Additionally, the actuator model has been used to account to remove the effect on the tracking error. Therefore, the effect of the actuator and control allocation dynamics on the tracking error is removed by the filter

$$
\dot{\Xi}_{n}=-C_{n} \Xi_{n}+\left(h(t, x, u, \hat{\theta})-\tau_{d}\right)
$$

The matrix $\Gamma_{\mathrm{ca}}$ can be time-varying to speed up convergence. For example if $\Gamma_{\mathrm{ca}}=\gamma\left(H^{T} H\right)^{-1}$ for some $\gamma>0$, then

$$
\left[\begin{array}{c}
\dot{u}_{d} \\
\dot{\lambda}
\end{array}\right]=-\gamma H^{-1}\left[\begin{array}{c}
\frac{\partial L}{\partial u_{d}} \\
\frac{\partial L}{\partial \lambda}
\end{array}\right]-H^{-1} u_{\mathrm{ff}}
$$

where the first term is the Newton direction when $L$ is considered the cost function to be minimized. In the case $H^{T} H$ is poorly conditioned, the choice $\Gamma_{\text {ca }}=\gamma\left(H^{T} H+\varsigma I\right)^{-1}$ for a small $\varsigma>0$ can be made. Using the design (9), (13), (14), the OCLF derivative becomes

$$
\dot{V}_{\mathrm{ca}}=-\sigma \sum_{i=1}^{n} \bar{z}_{i}^{T} C_{i} \bar{z}_{i}-\left[\begin{array}{c}
\frac{\partial L}{\partial u_{d}} \\
\frac{\partial L}{\partial \lambda}
\end{array}\right]^{T} H \Gamma_{\mathrm{ca}} H\left[\begin{array}{c}
\frac{\partial L}{\partial u_{d}} \\
\frac{\partial L}{\partial \lambda}
\end{array}\right],
$$

which shows that the set $\mathcal{A}$ is UGAS and therefore the augmented tracking error will converge to zero when $t \rightarrow \infty$. For appropriate choices of the virtual command filters, controller gains, and within the set of constraints on $x$ and $u, \Xi$ is small and therefore the tracking error $z_{1}$ converges to a small neighborhood around zero. The first order partial derivatives of the cost function with respect the desired control commands and the Lagrangian parameter converge to zero, and due to the cost function being convex, the desired commands and Lagrangian parameter converge to the optimal solution.

\section{Adaptive Control Design}

So far, it has been assumed in the design that the parameter $\theta$ was known. If the parameter $\theta$ is (partially) unknown, the control design from the previous section is not implementable. Hence, an estimate $\hat{\theta}$ and corresponding update law are introduced. Three different adaptation laws are presented, in the first the parameter update is designed simultaneously with the control laws: the integrated design. In the second design, modularity between the control laws and the identifier is achieved by means of introducing additional nonlinear damping terms, allowing non Lyapunov based identifiers to be applied. Finally, a hybrid combination of the two former designs is proposed which has better convergence properties as it uses information from both sources to update the estimate, and does not require nonlinear damping terms.

\section{IV.A. Integrated Update Law}

The Lyapunov-based update laws for the integrated adaptive design method are designed by extending the OCLF (11) with additional terms that penalize the parameter estimation error, yielding an adaptive optimizing CLF (AOCLF)

$$
V_{\mathrm{int}}=V_{\mathrm{ca}}+\frac{1}{2} \operatorname{tr}\left(\tilde{\theta}^{T} \Gamma_{\theta}^{-1} \tilde{\theta}\right)
$$

where $\Gamma_{\theta}^{T}=\Gamma_{\theta}>0$ is the update gain matrix, and $\tilde{\theta}=\theta-\hat{\theta}$. In this case, the task is to stabilize the set $\mathcal{A}_{\text {int }}$ defined by

$$
\mathcal{A}_{\text {int }}=\left\{\eta \in \mathbb{R}^{q} \mid Q(\eta)=0\right\}
$$

where $\eta=\left(\bar{z}^{T} \tilde{\theta}^{T} u_{d}^{T} \lambda^{T}\right)^{T}$, and $Q(\eta)=\left(\bar{z}^{T} \tilde{\theta}^{T} \frac{\partial L^{T}}{\partial u_{d}} \frac{\partial L^{T}}{\partial \lambda}\right)^{T}$. Taking the time derivative of (17), results in

$$
\begin{aligned}
\dot{V}_{\text {int }}= & -\sigma \sum_{i=1}^{n} \bar{z}_{i}^{T} C_{i} \bar{z}_{i}-\left[\begin{array}{c}
\frac{\partial L}{\partial u_{d}} \\
\frac{\partial L}{\partial \lambda}
\end{array}\right]^{T} H \Gamma_{\mathrm{ca}} H\left[\begin{array}{c}
\frac{\partial L}{\partial u_{d}} \\
\frac{\partial L}{\partial \lambda}
\end{array}\right]-\operatorname{tr}\left(\tilde{\theta}^{T} \Gamma_{\theta}^{-1} \dot{\hat{\theta}}\right)+\sigma \sum_{i=1}^{n}\left(\bar{z}_{i}^{T} \varphi_{i}^{T} \tilde{\theta}\right) \\
& +\left(\frac{\partial L^{T}}{\partial u_{d}} \frac{\partial^{2} L}{\partial x \partial u_{d}}+\frac{\partial L^{T}}{\partial \lambda} \frac{\partial^{2} L}{\partial x \partial \lambda}\right) \varphi_{u}^{T} \tilde{\theta}+\left(\frac{\partial L^{T}}{\partial u_{d}} \frac{\partial^{2} L}{\partial \hat{\theta} \partial u_{d}}+\frac{\partial L^{T}}{\partial \lambda} \frac{\partial^{2} L}{\partial \hat{\theta} \partial \lambda}\right) \dot{\hat{\theta}}
\end{aligned}
$$


where the parameter update law is used to cancel the indefinite parts, and the control allocation update law feed-forward signal now includes the parameter estimate update:

$$
\begin{aligned}
\dot{\hat{\theta}}= & \Gamma_{\theta}\left(\sigma \sum_{i=1}^{n} \varphi_{i} \bar{z}_{i}+\varphi_{u}\left(\frac{\partial^{2} L^{T}}{\partial x \partial u_{d}} \frac{\partial L}{\partial u_{d}}+\frac{\partial^{2} L^{T}}{\partial x \partial \lambda} \frac{\partial L}{\partial \lambda}\right)\right) \\
{\left[\begin{array}{c}
\dot{u}_{d} \\
\dot{\lambda}
\end{array}\right]=} & -\Gamma_{\mathrm{ca}} H\left[\begin{array}{c}
\frac{\partial L}{\partial u_{d}} \\
\frac{\partial L}{\partial \lambda}
\end{array}\right]-H^{-1} u_{\mathrm{ff}}, \\
u_{\mathrm{ff}}= & {\left[\begin{array}{c}
\frac{\partial^{2} L}{\partial t \partial u_{d}} \\
\frac{\partial^{2} L}{\partial t \partial \lambda}
\end{array}\right]+\left[\begin{array}{c}
\frac{\partial^{2} L}{\partial \bar{z} L u_{d}} \\
\frac{\partial^{2} L}{\partial \bar{z} \partial \lambda}
\end{array}\right]\left(G(t, x, u, \hat{\theta})-\dot{y}_{r}-\dot{\Xi}\right) } \\
& +\left[\begin{array}{c}
\frac{\partial^{2} L}{\partial \hat{\theta} \partial u_{d}} \\
\frac{\partial^{2} L}{\partial \hat{\theta} \partial \lambda}
\end{array}\right] \dot{\hat{\theta}}
\end{aligned}
$$

This choice of update laws results in the AOCLF time derivative

$$
\dot{V}_{\mathrm{int}}=-\sigma \sum_{i=1}^{n} \bar{z}_{i}^{T} C_{i} \bar{z}_{i}-\left[\begin{array}{c}
\frac{\partial L}{\partial u_{d}} \\
\frac{\partial L}{\partial \lambda}
\end{array}\right]^{T} H \Gamma_{\mathrm{ca}} H\left[\begin{array}{c}
\frac{\partial L}{\partial u_{d}} \\
\frac{\partial L}{\partial \lambda}
\end{array}\right],
$$

which shows UGS of the set $\mathcal{A}_{\text {int }}$. From LaSalle-Yoshizawa's theorem ${ }^{17}$ it follows that when $t \rightarrow \infty$, all solutions converge to the manifold $\left[\bar{z}^{T} \frac{\partial L^{T}}{\partial u_{d}} \frac{\partial L^{T}}{\partial \lambda}\right]^{T}=0$. It is possible to conclude UGAS of $\mathcal{A}_{\text {int }}$ for the integrated design when certain PE conditions are satisfied, and then demonstrating that the estimation error $\varphi^{T} \tilde{\theta}$ converges to zero, see e.g. ${ }^{18,19}$

\section{IV.B. Modular Update Law}

The modular adaptive design aims to separate the identifier from the the Lyapunov design, to allow nonLyapunov based parameter update laws. In order to achieve this for general nonlinear systems, nonlinear damping terms are required to make the control design robust against parameter estimation errors. In this case, these nonlinear damping terms are required both in the high level design and the control allocation update laws. First of all swapping filters are introduced since the state derivatives are, in general, not measurable or directly available. Two filters and a static mapping are introduced

$$
\begin{aligned}
\dot{\Omega}_{0} & =A\left(\Omega_{0}+x\right)-f(t, x, u) \\
\dot{\Omega}^{T} & =A \Omega^{T}+F(t, x, u)
\end{aligned}
$$

where

$$
\begin{aligned}
f(t, x, u) & =\left[\begin{array}{c}
f_{1}(t, x, u)+g_{1}(t, x, u) x_{2} \\
\vdots \\
f_{n}(t, x)+g_{n}(t, x) \varphi_{0}^{T}(t, x, u)
\end{array}\right], \\
F(t, x, u) & =\left[\begin{array}{c}
\varphi_{1}^{T}(t, x, u) \\
\vdots \\
\varphi_{n}^{T}(t, x)+g_{n}(t, x) \varphi_{u}^{T}(t, x, u)
\end{array}\right]
\end{aligned}
$$

and the estimation error is defined as

$$
\epsilon=\left(\Omega_{0}+x\right)-\Omega^{T} \hat{\theta}=\Omega^{T} \tilde{\theta}+\tilde{\epsilon}
$$

with $\tilde{\epsilon}=\left(\Omega_{0}+x\right)-\Omega^{T} \theta$. The matrix $A$ is negative definite, and strengthened

$$
A(t, x)=A_{0}-\rho F(t, x, u)^{T} F(t, x, u) P
$$

where $\rho>0$, and $P$ is a constant matrix such that

$$
P A_{0}+A_{0}^{T} P=-I, P=P^{T}>0 .
$$


This allows the following update laws to be used, which guarantee boundedness of the estimation error

$$
\dot{\hat{\theta}}=\Gamma_{\theta} \Omega \epsilon
$$

for a gradient based update law with $\Gamma_{\theta}$ a positive definite, symmetric matrix, and

$$
\begin{aligned}
\dot{\hat{\theta}} & =\Gamma_{\theta} \Omega \epsilon \\
\dot{\Gamma}_{\theta} & =-\Gamma_{\theta} \Omega \Omega^{T} \Gamma_{\theta}
\end{aligned}
$$

for a least-squares update law. Additionally, the high-level virtual control laws and control allocation update laws are augmented with nonlinear damping terms to achieve the desired separation. The virtual control laws become

$$
\alpha_{i}=g_{i}^{-1}\left(-f_{i}-\varphi_{i}^{T} \hat{\theta}-C_{i} z_{i}-S_{i} \bar{z}_{i}-g_{i-1}^{T} \bar{z}_{i-1}+\dot{x}_{i_{c}}\right)
$$

where the nonlinear damping matrices $S_{i}$ are defined as

$$
S_{i}=\varphi_{i}^{T} \kappa_{i} \varphi_{i}
$$

and $\kappa_{i}=\kappa_{i}^{T}>0$ are the nonlinear damping gain matrices of size $\mathbb{R}^{(p \times p)}$. Additional nonlinear damping is added in the design of the desired virtual control effort $\tau_{d}$, such that $S_{n}$ becomes

$$
S_{n}=\varphi_{i}^{T} \kappa_{i} \varphi_{i}+\varphi_{u}^{T} \kappa_{u} \varphi_{u}
$$

The update laws for control allocation become

$$
\left[\begin{array}{c}
\dot{u}_{d} \\
\dot{\lambda}
\end{array}\right]=-\Gamma_{\mathrm{ca}} H\left[\begin{array}{c}
\frac{\partial L}{\partial u_{d}} \\
\frac{\partial L}{\partial \lambda}
\end{array}\right]-H^{-1}\left(u_{\mathrm{ff}}+u_{\kappa}\right)
$$

where $u_{\mathrm{ff}}$ is the same as for the non-adaptive control design, and $u_{\kappa}$ is the contribution due to nonlinear damping defined as

$$
u_{\kappa}=\left[\begin{array}{c}
\frac{\partial^{2} L}{\partial x \partial u_{d}} \\
\frac{\partial^{2} L}{\partial x \partial \lambda}
\end{array}\right]^{T} \varphi_{u}^{T} \kappa_{u_{d}} \varphi_{u}\left[\begin{array}{c}
\frac{\partial^{2} L}{\partial x \partial u_{d}} \\
\frac{\partial^{2} L}{\partial x \partial \lambda}
\end{array}\right]\left[\begin{array}{c}
\frac{\partial L}{\partial u_{d}} \\
\frac{\partial L}{\partial \lambda}
\end{array}\right] .
$$

Using the designed update laws for the parameter estimate, the high-level control laws, and the control allocation update laws, ISS of the set $\mathcal{A}_{\text {mod }}=\mathcal{A}$ with respect to the parameter estimation error and its derivative is shown by means of the following OCLF

$$
V_{\text {mod }}=V_{\text {ca }} .
$$

The derivative of the OCLF along the trajectories of the system (1), the virtual control laws (31), and the control allocation update laws (34) becomes

$$
\begin{aligned}
\dot{V}_{\text {mod }}= & -\sigma \sum_{i=1}^{n} \bar{z}_{i}^{T} C_{i} \bar{z}_{i}-\sigma \sum_{i=1}^{n} \bar{z}_{i}^{T} S_{i} \bar{z}_{i}+\sigma \sum_{i=1}^{n}\left(\bar{z}_{i}^{T} \varphi_{i}^{T} \tilde{\theta}\right) \\
& +\left(\frac{\partial L^{T}}{\partial u_{d}} \frac{\partial^{2} L}{\partial x \partial u_{d}}+\frac{\partial L^{T}}{\partial \lambda} \frac{\partial^{2} L}{\partial x \partial \lambda}\right) \varphi_{u}^{T} \tilde{\theta}+\left(\frac{\partial L^{T}}{\partial u_{d}} \frac{\partial^{2} L}{\partial \hat{\theta} \partial u_{d}}+\frac{\partial L^{T}}{\partial \lambda} \frac{\partial^{2} L}{\partial \hat{\theta} \partial \lambda}\right) \dot{\hat{\theta}} \\
& -\left[\begin{array}{c}
\frac{\partial L}{\partial u_{d}} \\
\frac{\partial L}{\partial \lambda}
\end{array}\right]^{T} H \Gamma_{\text {ca }} H\left[\begin{array}{c}
\frac{\partial L}{\partial u_{d}} \\
\frac{\partial L}{\partial \lambda}
\end{array}\right]-\left[\begin{array}{c}
\frac{\partial L}{\partial u_{d}} \\
\frac{\partial L}{\partial \lambda}
\end{array}\right]^{T} u_{\kappa} \\
= & -\sigma \sum_{i=1}^{n} \bar{z}_{i}^{T} C_{i} \bar{z}_{i}-\sigma \sum_{i=1}^{n}\left(\varphi_{i} \bar{z}_{i}-\frac{1}{2} \kappa_{i}^{-1} \tilde{\theta}\right)^{T} \kappa_{i}\left(\varphi_{i} \bar{z}_{i}-\frac{1}{2} \kappa_{i}^{-1} \tilde{\theta}\right)
\end{aligned}
$$




$$
\begin{aligned}
& +\frac{\sigma}{4} \sum_{i=1}^{n} \tilde{\theta}^{T} \kappa_{i}^{-1} \tilde{\theta}-\sigma\left(\varphi_{u} \bar{z}_{n}-\frac{1}{2} \kappa_{u}^{-1} \tilde{\theta}\right)^{T} \kappa_{u}\left(\varphi_{u} \bar{z}_{n}-\frac{1}{2} \kappa_{u}^{-1} \tilde{\theta}\right) \\
& +\frac{\sigma}{4} \tilde{\theta}^{T} \kappa_{u}^{-1} \tilde{\theta}-\left[\begin{array}{c}
\frac{\partial L}{\partial u_{d}} \\
\frac{\partial L}{\partial \lambda}
\end{array}\right]^{T} H \Gamma_{\mathrm{ca}} H\left[\begin{array}{c}
\frac{\partial L}{\partial u_{d}} \\
\frac{\partial L}{\partial \lambda}
\end{array}\right] \\
& -\left(\varphi_{u}\left[\begin{array}{c}
\frac{\partial^{2} L}{\partial x \partial u_{d}} \\
\frac{\partial^{2} L}{\partial x \partial \lambda}
\end{array}\right]^{T}\left[\begin{array}{c}
\frac{\partial L}{\partial u_{d}} \\
\frac{\partial L}{\partial \lambda}
\end{array}\right]-\frac{1}{2} \kappa_{u_{d}}^{-1} \tilde{\theta}\right)^{T} \kappa_{u_{d}}\left(\varphi_{u}\left[\begin{array}{c}
\frac{\partial^{2} L}{\partial x \partial u_{d}} \\
\frac{\partial^{2} L}{\partial x \partial \lambda}
\end{array}\right]^{T}\left[\begin{array}{c}
\frac{\partial L}{\partial u_{d}} \\
\frac{\partial L}{\partial \lambda}
\end{array}\right]-\frac{1}{2} \kappa_{u_{d}}^{-1} \tilde{\theta}\right) \\
& +\frac{1}{4} \tilde{\theta}^{T} \kappa_{u_{d}}^{-1} \tilde{\theta} \\
& \leq-\sigma \sum_{i=1}^{n} \bar{z}_{i}^{T} C_{i} \bar{z}_{i}+\frac{\sigma}{4} \sum_{i=1}^{n} \tilde{\theta}^{T} \kappa_{i}^{-1} \tilde{\theta}+\frac{\sigma}{4} \tilde{\theta}^{T} \kappa_{u}^{-1} \tilde{\theta}+\frac{1}{4} \tilde{\theta}^{T} \kappa_{u_{d}}^{-1} \tilde{\theta} \\
& -\left[\begin{array}{c}
\frac{\partial L}{\partial u_{d}} \\
\frac{\partial L}{\partial \lambda}
\end{array}\right]^{T} H \Gamma_{\mathrm{ca}} H\left[\begin{array}{c}
\frac{\partial L}{\partial u_{d}} \\
\frac{\partial L}{\partial \lambda}
\end{array}\right]
\end{aligned}
$$

From the last inequality, a positively invariant compact set can be derived to which the augmented tracking errors and control allocation states will exponentially converge. From (37)

$$
\sigma c_{0} \bar{z}^{T} \bar{z}+\gamma_{0}\left(\frac{\partial L^{T}}{\partial u_{d}} \frac{\partial L}{\partial u_{d}}+\frac{\partial L^{T}}{\partial \lambda} \frac{\partial L}{\partial \lambda}\right) \geq \frac{1}{4} \tilde{\theta}^{T} \kappa_{0}^{-1} \tilde{\theta}
$$

where $c_{0}=\min _{i} \lambda_{\min }\left(C_{i}\right) \geq 0, \gamma_{0}=\inf _{t} \lambda_{\min }\left(H \Gamma_{\mathrm{ca}} H\right), \kappa_{0}^{-1}=\sigma \sum_{i=1}^{n} \kappa_{i}^{-1}+\sigma \kappa_{u}^{-1}+\kappa_{u_{d}}^{-1}$ implies that $\dot{V} \leq 0$. Hence, the inequality therefore describes an upper bound on the invariant compact set. ISS-stability of the set $\mathcal{A}_{\text {mod }}$ can be shown using an ISS-Lyapunov function since existence of such a function is equivalent to the system being ISS, ${ }^{17,20}$ if $\mathcal{A}_{\text {mod }}$ is compact, and 0-invariant.

\section{IV.C. Composite Update Law}

The designs above can be combined, having the benefit that the nonlinear damping terms are no longer required for stability, and faster convergence of the tracking errors as well as estimation error can be achieved. The composite design makes use of the nonlinear swapping filters (24), and has a composite update law defined as

$$
\dot{\hat{\theta}}=\Gamma_{\theta}\left(\sigma \sum_{i=1}^{n} \varphi_{i} \bar{z}_{i}+\varphi_{u}\left(\frac{\partial^{2} L^{T}}{\partial x \partial u_{d}} \frac{\partial L}{\partial u_{d}}+\frac{\partial^{2} L^{T}}{\partial x \partial \lambda} \frac{\partial L}{\partial \lambda}\right)+\Omega \Psi \epsilon\right)
$$

with $\Psi=\Psi^{T}>0$ a weight matrix to control how the adaptation law should weight information form the tracking error relative to the information coming from the estimation error. The update gain matrix $\Gamma_{\theta}$ can be kept constant for a gradient approach or updated through the weighted least-squares update law

$$
\dot{\Gamma}_{\theta}=-\Gamma_{\theta} \Omega \Psi \Omega^{T} \Gamma_{\theta} .
$$

The control allocation update laws are identical to the ones in the integrated adaptive design. The AOCLF from the integrated design is augmented with a term penalizing the residual error $\tilde{\epsilon}=\Omega_{0}+x-\Omega^{T} \theta$,

$$
V_{\text {comp }}=V_{\mathrm{int}}+\frac{1}{2} \tilde{\epsilon}^{T} P \Psi \tilde{\epsilon}
$$

This AOCLF will be used to show UGS of the set

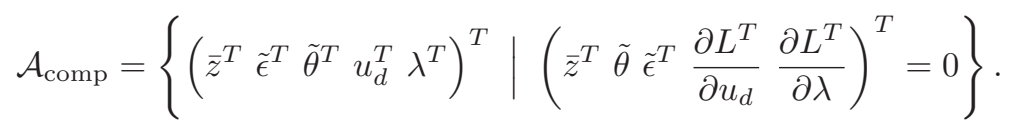


The time derivative of $V_{\text {comp }}$ along the solutions of the system, with the integrated control laws and composite update laws becomes

$$
\begin{aligned}
\dot{V}_{\text {comp }}= & -\sigma \sum_{i=1}^{n} \bar{z}_{i}^{T} C_{i} \bar{z}_{i}-\frac{1}{2} \epsilon^{T} \Psi \Omega^{T} \tilde{\theta}-\frac{1}{2} \tilde{\theta}^{T} \Omega \Psi \tilde{\epsilon}+\frac{1}{2}\left(\dot{\tilde{\epsilon}}^{T} P \Psi \tilde{\epsilon}+\tilde{\epsilon}^{T} \Psi P \dot{\tilde{\epsilon}}\right) \\
& -\left[\begin{array}{c}
\frac{\partial L}{\partial u_{d}} \\
\frac{\partial L}{\partial \lambda}
\end{array}\right]^{T} H \Gamma_{\mathrm{ca}} H\left[\begin{array}{c}
\frac{\partial L}{\partial u_{d}} \\
\frac{\partial L}{\partial \lambda}
\end{array}\right] \\
\leq & -\sigma \sum_{i=1}^{n} \bar{z}_{i}^{T} C_{i} \bar{z}_{i}-\frac{1}{2} \epsilon^{T} \Psi \epsilon+\frac{1}{2} \tilde{\epsilon} \Psi \tilde{\epsilon}-\frac{1}{2} \tilde{\epsilon} \Psi \tilde{\epsilon}-\left[\begin{array}{c}
\frac{\partial L}{\partial u_{d}} \\
\frac{\partial L}{\partial \lambda}
\end{array}\right]^{T} H \Gamma_{\mathrm{ca}} H\left[\begin{array}{c}
\frac{\partial L}{\partial u_{d}} \\
\frac{\partial L}{\partial \lambda}
\end{array}\right] \\
= & -\sigma \sum_{i=1}^{n} \bar{z}_{i}^{T} C_{i} \bar{z}_{i}-\frac{1}{2} \epsilon^{T} \Psi \epsilon-\left[\begin{array}{c}
\frac{\partial L}{\partial u_{d}} \\
\frac{\partial L}{\partial \lambda}
\end{array}\right]^{T} H \Gamma_{\mathrm{ca}} H\left[\begin{array}{c}
\frac{\partial L}{\partial u_{d}} \\
\frac{\partial L}{\partial \lambda}
\end{array}\right]
\end{aligned}
$$

showing UGS of the set $\mathcal{A}_{\text {comp }}$. The fact that the dynamics of $\tilde{\epsilon}$ are governed by $\dot{\tilde{\epsilon}}=A$

tilde€ has been used to provide an upper bound on part of the AOCLF time derivative. From the LaSalleYoshizawa theorem it follows that when $t \rightarrow \infty$, all solutions converge to the manifold $\left[\bar{z}^{T} \epsilon^{T} \frac{\partial L^{T}}{\partial u_{d}} \frac{\partial L^{T}}{\partial \lambda}\right]^{T}=$ 0 . Note that the major difference between the integrated and composite designs is that in the composite design also convergence of the estimation error to zero is guaranteed. Convergence of the parameter estimation error $\tilde{\theta}$ to zero can only be guaranteed when a PE condition is satisfied.

\section{Fighter Aircraft Model}

The relatively simple, yet nonlinear, aircraft model presented in Ref. 1 is selected as a suitable model to compare the control designs. The aircraft model, described by Eqs. (44), has resemblances to an F-18 aircraft model. Aerodynamic data is available in the form of tables for two trimmed flight conditions: one at an altitude of $30000 \mathrm{ft}$ and Mach number 0.7, the other at $40000 \mathrm{ft}$ and a Mach number of 0.6. The model has seven independent control surfaces: left and right horizontal stabilizers, left and right ailerons, collective leading edge flaps, collective trailing edge flaps, and collective rudder. A layout of the aircraft and its control surfaces is shown in Fig. 1. The main simplifications made to derive the dynamic model are the constant airspeed assumption, and secondly, no lift and drag effects on the control surfaces. The last assumption was made to transform the system into lower triangular form such that standard FBL/NDI, or (adaptive) backstepping can be applied. The designs considered in this paper do not suffer from this limitation due to the use of the filters to generate the intermediate control laws. ${ }^{21}$ The aircraft model includes second order actuator dynamics. The magnitude, rate, and bandwidth limits of the various actuators are specified in Table 1.

$$
\begin{aligned}
\frac{d}{d t}\left(\begin{array}{c}
\alpha \\
\beta \\
\phi \\
\theta \\
p \\
q \\
r
\end{array}\right)= & \left(\begin{array}{c}
q-p \beta+z_{\alpha} \Delta \alpha+\left(g_{0} / V\right)\left(\cos \vartheta \cos \phi-\cos \vartheta_{0}\right) \\
y_{\beta}+p\left(\sin \alpha_{0}+\Delta \alpha\right)-r \cos \alpha_{0}+\left(g_{0} / V\right) \cos \vartheta \sin \phi \\
p+q \tan \vartheta \sin \phi+r \tan \vartheta \cos \phi \\
q \cos \phi-r \sin \theta \\
l_{\beta} \beta+l_{q} q+l_{r} r+\left(l_{\beta \alpha} \beta+l_{r \alpha} r\right) \Delta+l_{p} p-i_{1} q r \\
m_{\alpha} \Delta \alpha+m_{q} q+i_{2} p r-m_{\dot{\alpha}}\left(g_{0} / V\right)\left(\cos \vartheta \cos \phi-\cos \vartheta_{0}\right) \\
n_{\beta} \beta+n_{r} r+n_{p} p+n_{p \alpha} p \Delta-i_{3} p q+n_{q} q
\end{array}\right) \\
& +\left(\begin{array}{ccccccc}
0 & 0 & 0 & 0 & 0 & 0 & 0 \\
0 & 0 & 0 & 0 & 0 & 0 & 0 \\
0 & 0 & 0 & 0 & 0 & 0 & 0 \\
0 & 0 & 0 & 0 & 0 & 0 & 0 \\
l_{\delta_{e l}} & l_{\delta_{e r}} & l_{\delta_{a l}} & l_{\delta_{a r}} & 0 & 0 & l_{\delta_{r}} \\
m_{\delta_{e l}} & m_{\delta_{e r}} & m_{\delta_{a l}} & m_{\delta_{a r}} & m_{\delta_{l e f}} & m_{\delta_{t e f}} & m_{\delta_{r}} \\
n_{\delta_{e l}} & n_{\delta_{e r}} & n_{\delta_{a l}} & n_{\delta_{a r}} & 0 & 0 & n_{\delta_{r}}
\end{array}\right)\left(\begin{array}{c}
\delta_{e l} \\
\delta_{e r} \\
\delta_{a l} \\
\delta_{a r} \\
\delta_{l e f} \\
\delta_{t e f} \\
\delta_{r}
\end{array}\right)
\end{aligned}
$$




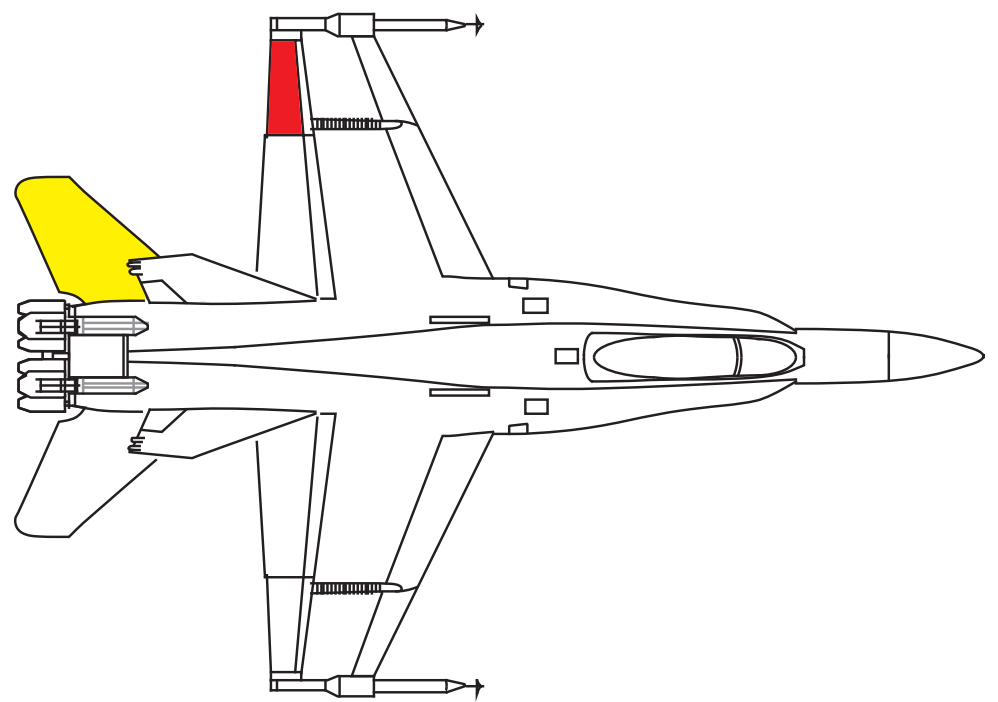

Figure 1. The control surfaces of the fighter aircraft model. The control surfaces which will lock in place during the various simulation scenarios are colored.

All stability and control derivatives introduced in Eqs. (44) are considered to be unknown and will be estimated on-line by the parameter estimation process of the adaptive control laws. The system Eqs. (44) is rewritten in a more suitable form for the control design as

$$
\begin{aligned}
& \dot{x}_{1}=f_{1}\left(x_{1}, x_{3}\right)+\varphi_{1}^{T}\left(x_{1}, x_{3}\right) \theta+g_{1}\left(x_{1}, x_{3}\right) x_{2} \\
& \dot{x}_{2}=f_{2}\left(x_{1}, x_{2}, x_{3}\right)+\varphi_{2}^{T}\left(x_{1}, x_{2}, x_{3}\right) \theta+g_{2}(\theta) u \\
& \dot{x}_{3}=f_{3}\left(x_{1}, x_{2}, x_{3}\right)
\end{aligned}
$$

where $x_{1}=\left[\begin{array}{lll}\phi & \alpha & \beta\end{array}\right]^{T}, x_{2}=\left[\begin{array}{lll}p & q & r\end{array}\right]^{T}, u=\left[\begin{array}{lllllll}\delta_{e l} & \delta_{e r} & \delta_{a l} & \delta_{a r} & \delta_{\text {lef }} & \delta_{\text {tef }} & \delta_{r}\end{array}\right]^{T}$ and the uncontrolled state $x_{3}=\vartheta$. The known nonlinear aircraft dynamics are represented by the vector functions $f_{1}\left(x_{1}, x_{3}\right), f_{2}\left(x_{1}, x_{2}, x_{3}\right)$ and $f_{3}\left(x_{1}, x_{2}, x_{3}\right)$ and the matrix function $g_{1}\left(x_{1}, x_{3}\right)$. The functions $\varphi_{1}\left(x_{1}, x_{3}\right)$ and $\varphi_{2}\left(x_{1}, x_{2}, x_{3}\right)$ are the regressor matrices, while $\theta$ and $g_{2}(\theta)$ are a vector and a matrix function containing the unknown parameters of the system, defined as

$$
\begin{aligned}
& \theta=\left[z_{\alpha}, y_{\beta}, l_{\beta}, l_{p}, l_{q}, l_{r}, l_{\beta \alpha}, l_{r \alpha}, l_{0}, m_{\alpha}, m_{q}, m_{\dot{\alpha}}, m_{0}, \ldots\right. \\
& \left.n_{\beta}, n_{p}, n_{q}, n_{r}, n_{p \alpha}, n_{0}\right]^{T} \\
& g_{2}(\theta)=\left[\begin{array}{ccccccc}
l_{\delta_{e l}} & l_{\delta_{e r}} & l_{\delta_{a l}} & l_{\delta_{a r}} & 0 & 0 & l_{\delta_{r}} \\
m_{\delta_{e l}} & m_{\delta_{e r}} & m_{\delta_{a l}} & m_{\delta_{a r}} & m_{\delta_{l e f}} & m_{\delta_{t e f}} & m_{\delta_{r}} \\
n_{\delta_{e l}} & n_{\delta_{e r}} & n_{\delta_{a l}} & n_{\delta_{a r}} & 0 & 0 & n_{\delta_{r}}
\end{array}\right]
\end{aligned}
$$

Note that the parameters $l_{0}, m_{0}$ and $n_{0}$ have been added to the vector $\theta$ to compensate for additional trim moments caused by locked actuators.

\section{V.A. Controller Design}

The control design follows the nominal backstepping design presented in Ref. 2 for the high level control design. This design is extended with dynamic control allocation and parameter estimation laws according to the composite adaptive design. The following cost function has been used

$$
J\left(u_{d}\right)=u_{d}^{T} W u_{d}+J_{2}\left(u_{d}\right)
$$

where $W=W^{T}>0$, and $J_{2}$ includes the magnitude constraints for this aircraft by means of barrier functions

$$
J_{2}\left(u_{d}\right)=-w \ln \left(u_{d}-u_{d, \min }\right)-w \ln \left(-u_{d}+u_{d, \max }\right)
$$


Table 1. Actuator Specifications.

\begin{tabular}{cccc}
\hline \hline Surface & $\begin{array}{c}\text { Deflection } \\
\text { Limit }[\mathrm{deg}]\end{array}$ & $\begin{array}{c}\text { Rate } \\
\text { Limit }[\mathrm{deg} / \mathrm{s}]\end{array}$ & $\begin{array}{c}\text { Bandwidth } \\
{[\mathrm{rad} / \mathrm{s}]}\end{array}$ \\
\hline Horizontal Stabilizer & {$[-24,10.5]$} & \pm 40 & 50 \\
Ailerons & {$[-25,45]$} & \pm 100 & 50 \\
Leading Edge Flaps & {$[-3,33]$} & \pm 15 & 50 \\
Trailing Edge Flaps & {$[-8,45]$} & \pm 18 & 50 \\
Rudder & {$[-30,30]$} & \pm 82 & 50 \\
\hline \hline
\end{tabular}

where $w>0$ is a tuning gain, $u_{d, \min }$ and $u_{d, \max }$ represent the lower and upper bound on $u_{d}$ respectively.

The numerical simulations have been performed in the MATLAB/Simulink environment, with an adaptive time-step, third-order accurate solver. The tuning parameters summarized in table 2 were used in the design. Additionally, covariance resetting was introduced to speed-up the estimation after a failure is detected. When a low-pass filtered version of the squared estimation error exceeds a certain threshold, and the covariance matrix is small, it is reset. Hence, the covariance matrix will only be reset if there is confidence in the estimated parameters, and there is a large estimation error.

Table 2. Adaptive Optimizing Controller Tuning Parameters

\begin{tabular}{|c|c|}
\hline Parameter & Value \\
\hline$C_{1}$ & $I_{3}$ \\
\hline$C_{2}$ & $2 I_{3}$ \\
\hline$S_{0}$ & $1 \times 10^{-1} I_{36}$ \\
\hline$\gamma$ & 500 \\
\hline$w$ & $0.1 \cdot \operatorname{diag}\left(\begin{array}{lllllll}1 & 1 & 1 & 1 & 1 & 1 & 1\end{array}\right)$ \\
\hline$W$ & $\operatorname{diag}\left(\begin{array}{lllllll}1 & 1 & 2 & 2 & 2 & 2 & 1\end{array}\right)$ \\
\hline$A_{0}$ & $-100 I_{6}$ \\
\hline$\rho$ & 10000 \\
\hline$\epsilon_{\min }$ & $1 \times 10^{-6}$ \\
\hline$\Gamma_{\text {reset }}$ & $10 S_{0} S_{0}$ \\
\hline$S_{\text {reset }}$ & $10 I_{36}$ \\
\hline$R$ & 1000 \\
\hline$\lambda$ & 0.005 \\
\hline
\end{tabular}

\section{V.B. Nominal Simulation Results}

Before considering actuator failures, the nominal simulation results are presented. The parameter vector is initialized with the values used in the model, hence, the on-board and the actual aircraft model are identical. The tracking performance for the aircraft performing three simultaneous angle of attack and bank angle doublets is shown in Fig. 2. The desired references are tracked nearly perfect, and the sideslip angle is kept very close to zero during the whole maneuver. Due to the aircraft model and on-board model being identical, the composite adaptive controller does not update the on-board model parameters. The commanded control deflections and the realized deflections are shown in Fig. 3. Note that for this controller tuning and reference signal the leading and trailing edge flaps contribute significantly to the realized control effort.

\section{V.C. Elevator Failure}

Several elevator lock-in-place failures have been investigated, only the severest considered failure is discussed here. After three seconds in the simulation, the left elevator deflects to -20 degrees and locks there. Hence, a trim moment is introduced, and the effectiveness of the left elevator seems zero. This failure is detected rapidly by the change detection mechanism, and the update gain matrix is reset. After the failure 

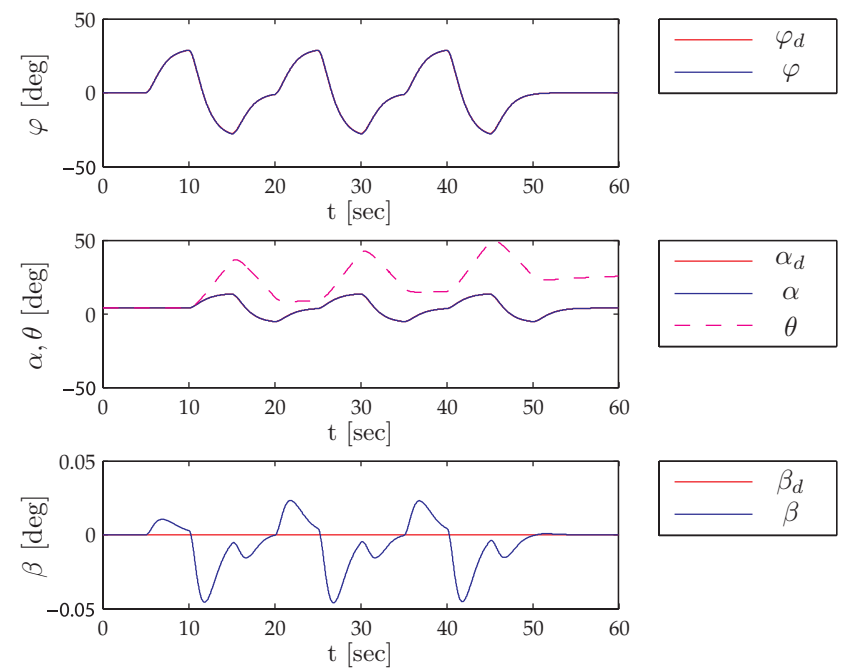

Figure 2. Tracking performance of the composite adaptive optimizing controller in the nominal case.
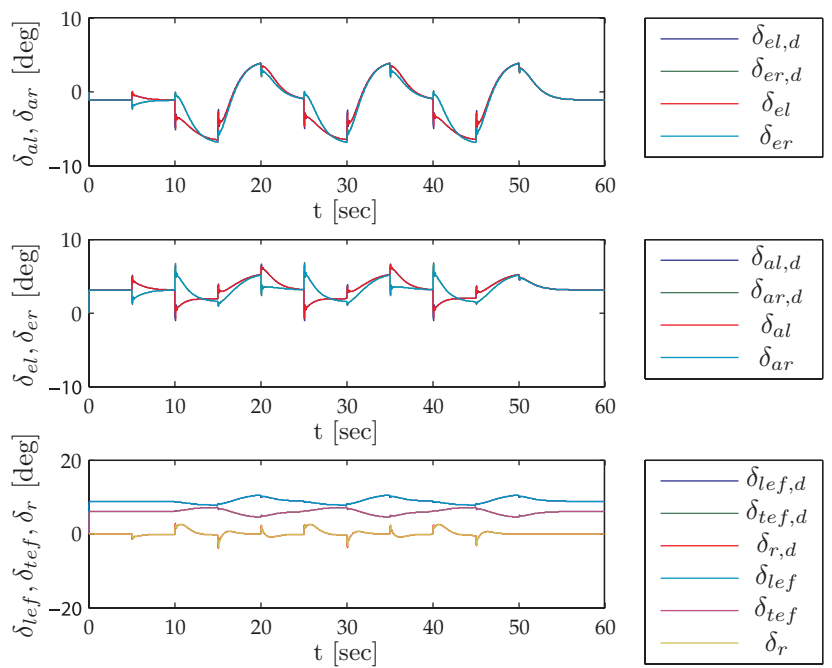

Figure 3. Control deflections commands and positions in the nominal case. 
is detected, the controller reconfigures very quickly and is able to complete the maneuver with excellent tracking performance as shown in Fig. 4. After the failure is injected and detected there are fast transients in the estimated parameters, as observed in Fig. 5. After the estimates have settled near their values sufficient for convergence of the augmented tracking error, they will start converging to their true values if a PE condition is satisfied. The control deflections used by the controller are shown in Fig. 6.
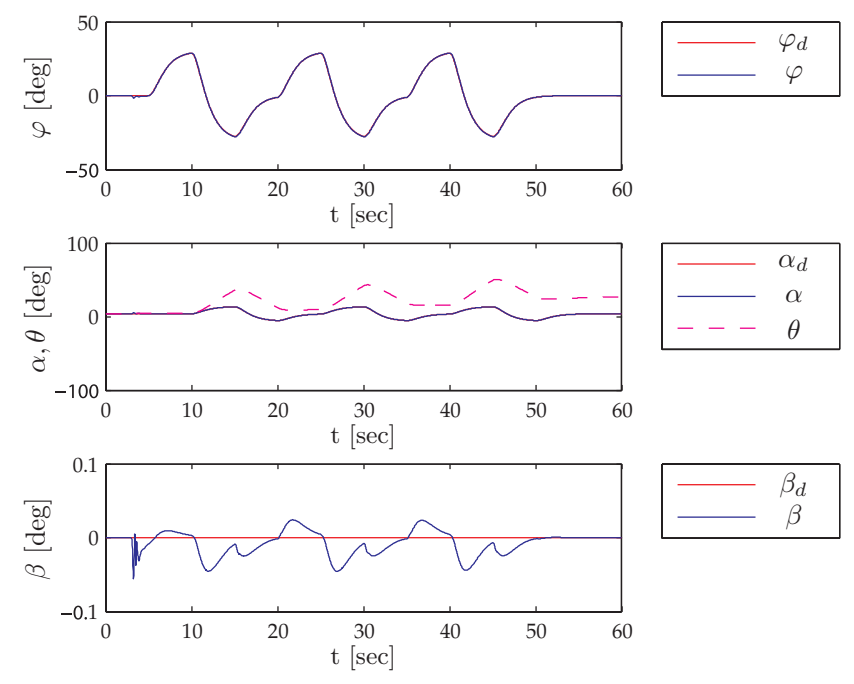

Figure 4. Tracking performance of the composite adaptive optimizing controller for a left elevator lock to -20 degrees after 3 seconds.
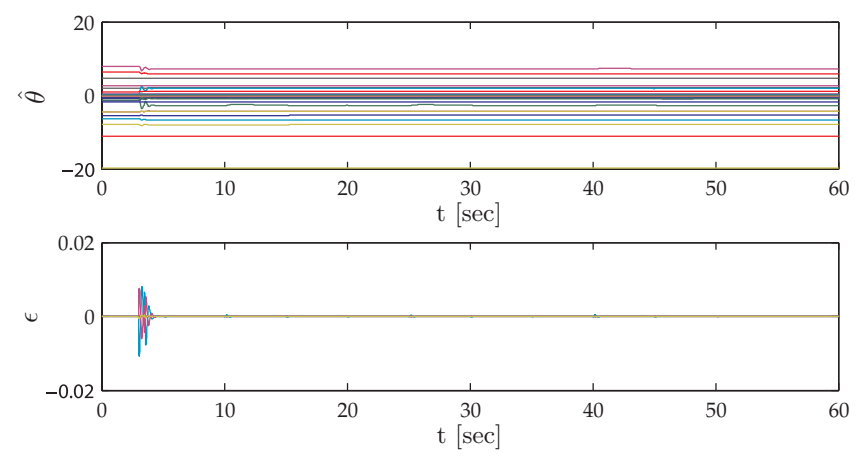

Figure 5. Parameter estimates and filtered estimation error for a left elevator lock to -20 degrees after 3 seconds.

\section{V.D. Aileron Failure}

Similar as for the elevator failures, aileron actuator failures have been investigated. Once again, only the worst case failure is discussed here. After three seconds into the maneuver, the left aileron deflects to 45 degrees trailing edge down. A transient can be observed from Fig. 7 after injection of the failure. The controller rapidly reconfigures and is able to track the reference commands very well. The failure is accommodated by asymmetric deflection of the elevators, with help from the remaining functional aileron as shown in Fig. 9. The parameter estimates and filtered estimation error are shown in Fig. 8, after initial transients the parameter estimates start converging to their true values and the filtered estimation error converges to zero. 

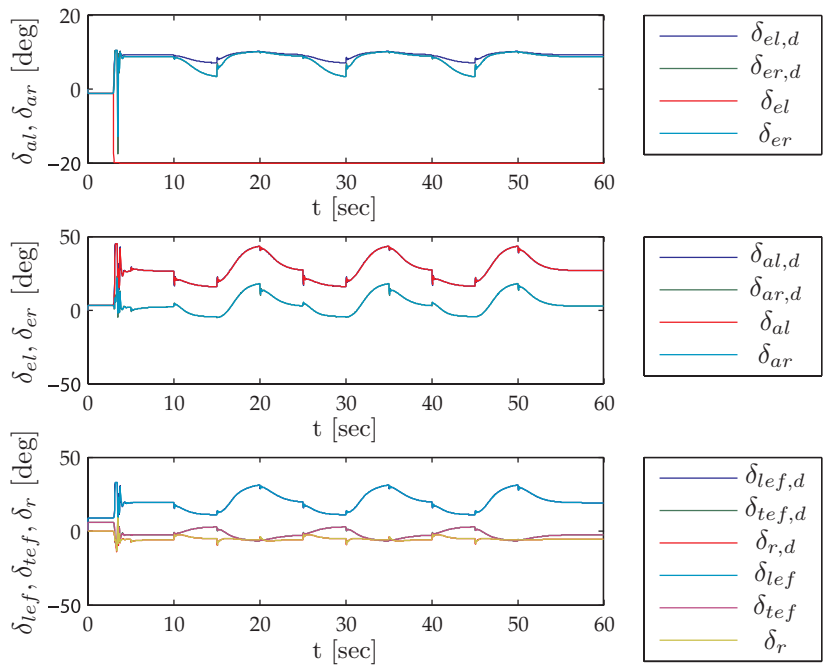

Figure 6. Control deflections commands and positions for a left elevator lock to -20 degrees after 3 seconds.
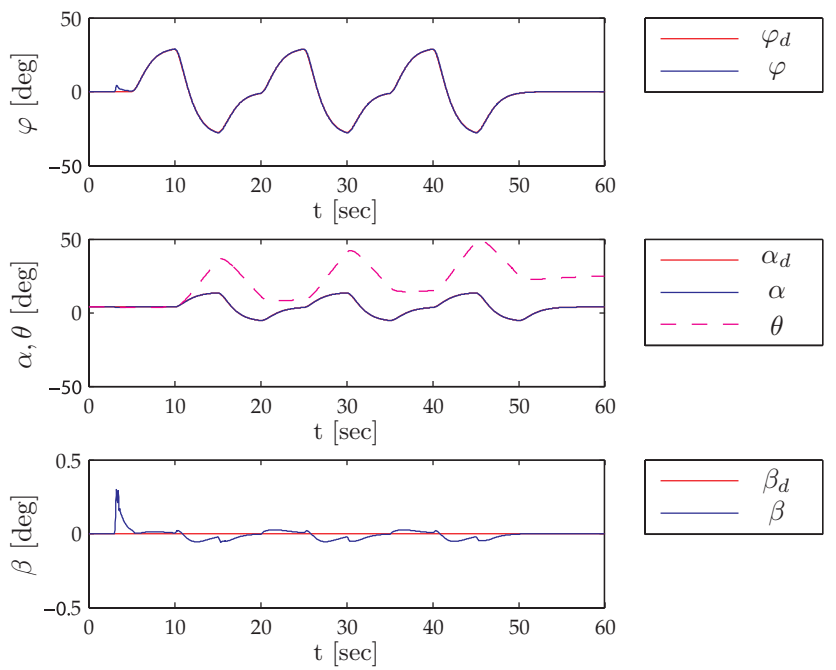

Figure 7. Tracking performance of the composite adaptive optimizing controller for a left aileron lock to 45 degrees after 3 seconds.
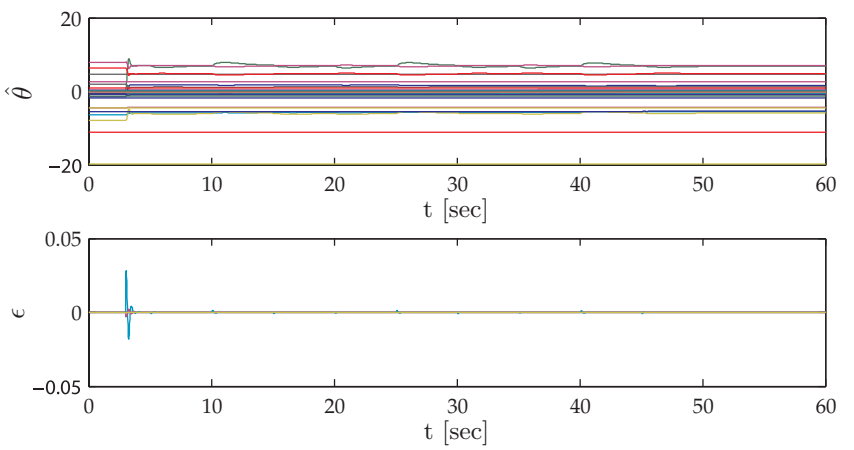

Figure 8. Parameter estimates and filtered estimation error for a left aileron lock to 45 degrees after 3 seconds.

14 of 16 

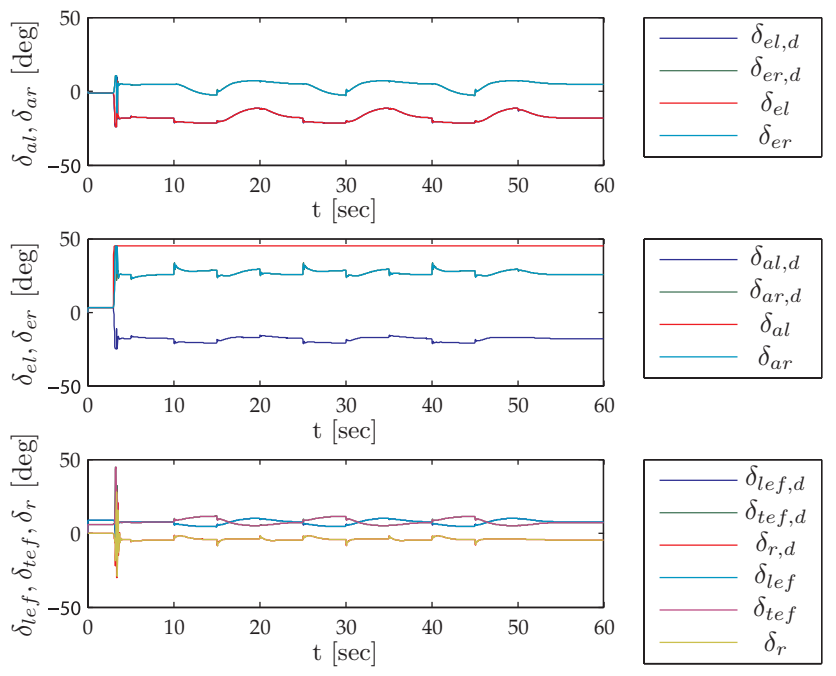

Figure 9. Control deflections commands and positions for a left aileron lock to 45 degrees after 3 seconds.

\section{Conclusions}

In this paper an adaptive control design is presented for over-actuated system and applied to a simple aircraft model. The control design has been separated into a high-level control law, dynamic control allocation update laws, and an parameter update law. Stability of the time-varying optimal sets has been proven through Lyapunov analysis. Closed loop boundedness of the tracking errors can be proven by means of AOCLFs. Since the state and control effectors of a system are generally bounded, locally asymptotic tracking of the desired reference signal can be concluded. The optimizing control allocation algorithm is also defined by means of a Lyapunov function, and it does not solve the optimization problem exactly at each step but rather (continuously) converges to the time varying optimal solution. The tracking performance of the adaptive control design is excellent for the nominal case and all considered failure cases. The failures are identified shortly after they are introduced into the system, and the new dynamics are rapidly identified. The composite adaptive design has the best convergence properties and does not require nonlinear damping to achieve this. The dynamic order of the resulting controller is equal to that of the modular adaptive design, and considerably higher than the integrated design due to inclusion of the update gain $\Gamma_{\theta}$ as states of the controller. The control allocation algorithm is not iterative, and does not depend on optimization software.

The proposed control scheme can be extended in different directions. Currently, it requires state measurements. In many applications (full) state measurements are not available, such that observers and estimators become necessary. This has to be accounted for in the control allocation design to guarantee boundedness of the tracking error. Extend the method to handle non-convex optimization problems. Considering antiwindup strategies, and possibly slack variables to enable faster convergence and handle solution infeasibility. Furthermore, inclusion of rate constraints on the control effectors. Actuator models can be included in the design, and actuator model parameters can be estimated on-line.

\section{References}

\footnotetext{
${ }^{1}$ Page, A. B. and Steinberg, M. L., "Effects of Control Allocation Algorithms on a Nonlinear Adaptive Design," Proceedings of the AIAA Guidance, Navigation, and Control Conference and Exhibit, Portland, OR, 81999.

${ }^{2}$ van Oort, E. R., Sonneveldt, L., Chu, Q. P., and Mulder, J. A., "A Comparison of Adaptive Nonlinear Control Designs for an Over-actuated Fighter Aircraft Model," AIAA Guidance, Navigation, and Control Conference and Exhibit, Honolulu, Hawaii, USA, 82008.

${ }^{3}$ Enns, D. F., "Control Allocation Approaches," Proceedings of the AIAA Guidance, Navigation, and Control Conference and Exhibit, Boston MA, USA, 1998, pp. 98-108.

${ }^{4}$ Buffington, J. M., Enns, D. F., and Teel, A. R., "Control Allocation and Zero Dynamics," Journal of Guidance, Control, and Dynamics, Vol. 21, No. 3, 1998, pp. 458-464.

${ }^{5}$ Sørdalen, O. J., "Optimal Thrust Allocation for Marine Vessels," Control Engineering Practice, Vol. 5, No. 9, 1997, pp. $1223-1231$.
} 
${ }^{6}$ Bodson, M., "Evaluation of Optimization Methods for Control Allocation," Journal of Guidance, Control, and Dynamics, Vol. 25, No. 4, 2002, pp. 703-711.

${ }^{7}$ Härkegård, O., Backstepping and Control Allocation with Applications to Flight Control, Ph.D. thesis, Linköping University, Linköping, Sweden, 2003.

${ }^{8}$ Luo, Y., Serrani, A., Yurkovich, S., Doman, D., and Oppenheimer, M., "Model Predictive Dynamic Control Allocation with Actuator Dynamics," Proceedings of the American Control Conference, Vol. 2, Boston, MA, USA, 2004, pp. 1695-1700.

${ }^{9}$ Luo, Y., Serrani, A., Yurkovich, S., Doman, D., and Oppenheimer, M., "Dynamic Control Allocation with Asymptotic Tracking of Time-varying Control Input Commands," Proceedings of the American Control Conference, Portland, OR, USA, 2005, pp. 2098-2103.

${ }^{10}$ Poonamallee, V., Yurkovich, S., Serrani, A., and Doman, D., "A Nonlinear Programming Approach for Control Allocation," Proceedings of the American Control Conference, Vol. 2, Boston, MA, USA, 2004, pp. 1689-1694.

${ }^{11}$ Johansen, T. A., Fossen, T. I., and Berge, S. P., "Constrained Nonlinear Control Allocation with Singularity Avoidance using Sequential Quadratic Programming," Control Systems Technology, IEEE Transactions on, Vol. 12, No. 1, 2004, pp. 211216.

${ }^{12}$ Johansen, T. A., Fossen, T. I., and Tøndel, P., "Efficient Optimal Constrained Control Allocation via Multi-parametric Programming," Journal of Guidance, Control, and Dynamics, Vol. 28, No. 3, 2005, pp. 506-515.

${ }^{13}$ Nocedal, J. and Wright, S. J., Numerical Optimization, chap. 12, Springer, 2nd ed., 2006, pp. 304-351.

${ }^{14}$ Sonneveldt, L., Chu, Q. P., and Mulder, J. A., "Nonlinear Flight Control Design using Constrained Adaptive Backstepping," Journal of Guidance, Navigation, and Dynamics, Vol. 30, No. 2, 2007, pp. 322-336.

${ }^{15}$ van Oort, E. R., Sonneveldt, L., Chu, Q. P., and Mulder, J. A., "Full Envelope Modular Adaptive Control of a Fighter Aircraft using Orthogonal Least Squares," Journal of Guidance, Navigation and Dynamics, Vol. 33, No. 5, 2010, pp. $1461-1472$.

${ }^{16}$ Farrell, J. A., Polycarpou, M., Sharma, M., and Dong, W., "Command Filtered Backstepping," Automatic Control, IEEE Transactions on, Vol. 54, No. 6, 6 2009, pp. 1391-1395. 1995.

${ }^{17}$ Krstić, M., Kanellakopoulos, I., and Kokotović, P. V., Nonlinear and Adaptive Control Design, John Wiley and Sons,

${ }^{18}$ Tjønnås, J., Nonlinear and Adaptive Dynamic Control Allocation, Ph.D. thesis, Norwegian University of Science and Technology, Faculty of Information Technology, Mathematics, and Electrical Engineering, Trondheim, Norway, 2008.

${ }^{19}$ Tjønnås, J. and Johansen, T. A., "Optimizing Nonlinear Adaptive Control Allocation," Proceedings of the 16th IFAC World Congress, Prague, Czcech Republic, 2005.

${ }^{20}$ Sontag, E. D. and Wang, Y., "On Characterizations of Input-to-State Stability with Respect to Compact Sets," Proceedings of the IFAC Non-linear Control Systems Design Symposium, Tahoe City, CA, USA, 1995, pp. 226-231.

${ }^{21}$ Farrell, J. A., Sharma, M., and Polycarpou, M., "Backstepping Based Flight Control with Adaptive Function Approximation," Journal of Guidance, Control and Dynamics, Vol. 28, No. 6, 1 2005, pp. 1089-1102. 